\title{
Urban-Rural Differences in Indiana Pediatric Firearm Trauma
}

\author{
Cory Wuerch ${ }^{1}$, Jodi Raymond, MPH, CSTR, CAISS ${ }^{2}$, Joseph O'Neil, MD, MPH², \\ FAAP, Matthew P. Landman, MD, MPH, FAAP, FACS ${ }^{2}$ \\ ${ }^{1}$ Indiana University School of Medicine, ${ }^{2}$ Riley Hospital for Children at Indiana \\ University Health
}

\begin{abstract}
Background and Hypothesis: Several studies have evaluated differences in firearm injuries among children and adolescents based on population. However, many of these studies exclude patients who die before arriving at a trauma center. We therefore hypothesize that important population-based differences in pediatric firearm injuries may be uncovered with inclusion of both pre-hospital firearm mortalities and patients treated at a tertiary children's hospital.
\end{abstract}

Methods: Patients less than 15 years of age who sustained a firearms-related injury/death between the years 2012 and 2018 were identified in: (1) death certificates from the Office of Vital Statistics State of Indiana and (2) Riley Hospital for Children Trauma Registry. Counties were classified as either urban, midsized, or rural based on the National Center for Health Statistic's population data. Bivariate analyses were used to analyze important variables with statistical significance set at $p<0.05$.

Results: A total of 222 patients were identified ( $77 \%$ male, mean age $=11.3 \pm$ 4.2 years). The median age of firearm injury survivors was 13 (IQR 7-14), while the median age of nonsurvivors was 14 (IQR 11-15). The county population was associated with injury intent, where the frequency of assaults/homicides was higher than expected in urban counties compared to midsized and rural counties $(p<0.05$ and $p<0.001$, respectively). Suicide frequency was higher than expected in rural counties $(p<0.001)$. Race was associated with injury intent $(p<0.001)$. Rural and midsized counties had higher than expected mortalities compared to urban counties ( $p<0.001$ and $p<0.05$, respectively). Attempted suicides in rural areas were more likely to be associated with mortality than attempts in urban areas $(p<0.001)$.

Conclusion and Potential Impact: Important differences exist between firearm injuries based on where they occur. The findings presented here will inform public health initiatives aimed at reducing firearm injury and death in Indiana. 